\title{
Relaxations and nano-phase-separation in ultraviscous heptanol-alkyl halide mixture
}

\author{
G. Power and J. K. Vij ${ }^{\text {a) }}$ \\ Laboratory of Advanced Electronic Materials, Department of Electronic Engineering, Trinity College, \\ University of Dublin, Dublin 2, Ireland \\ G. P. Johari \\ Department of Materials Science and Engineering, McMaster University, Hamilton, Ontario 5M4L7, \\ Canada
}

(Received 16 October 2006; accepted 20 November 2006; published online 19 January 2007)

\begin{abstract}
To gain insight into the effects of liquid-liquid phase separation on molecular relaxation behavior we have studied an apparently homogeneous mixture of 5-methyl-2-hexanol and isoamylbromide by dielectric spectroscopy over a broad temperature range. It shows two relaxation regions, widely separated in frequency and temperature, with the low-frequency relaxation due to the alcohol and the high-frequency relaxation due to the halide. In the mixture, the equilibrium dielectric permittivity $\varepsilon_{s}$ of the alcohol is $41 \%$ of the pure state at $155.7 \mathrm{~K}$ and $\varepsilon_{s}$ of isoamylbromide is $\sim 86 \%$ of the pure state at $128.7 \mathrm{~K}$. The difference decreases for the alcohol component with decreasing temperature and increases for the isoamylbromide component. The relaxation time $\tau$ of 5-methyl-2-hexanol in the mixture at $155.7 \mathrm{~K}$ is over five orders of magnitude less than in the pure state, and this difference increases with decreasing temperature, but $\tau$ of isoamylbromide in the mixture is marginally higher than in the pure liquid. This shows that the mixture would have two $T_{g}$ 's corresponding to its $\tau$ of $10^{3} \mathrm{~s}$, with values of $\sim 121 \mathrm{~K}$ for its 5 -methyl-2-hexanol component and $\sim 108 \mathrm{~K}$ for its isoamylbromide component. It is concluded that the mixture phase separates in submicron or nanometer-size aggregates of the alcohol in isoamylbromide, without affecting the latter's relaxation kinetics, while its own $\varepsilon_{s}$ and $\tau$ decrease markedly. (C) 2007 American Institute of Physics. [DOI: 10.1063/1.2409929]
\end{abstract}

\section{INTRODUCTION}

In one of the earliest single frequency dielectric studies, Schallamach ${ }^{1}$ had noted that 1-propanol isoamylbromide and geraniol isoamylbromide mixtures show two dielectric relaxation processes. As these features were similar to those observed in the pure liquids, he suggested that the relaxation features of a hydrogen-bonded liquid were not greatly affected by the presence of isoamylbromide, and "that a mixture of associated and nonassociated liquids is not microscopically homogeneous." ${ }^{11}$ Later, Denney ${ }^{2}$ briefly studied the dielectric relaxation spectra of 1-propanol isoamylbromide mixture and found that the relaxation time of 1-propanol in the mixture increased but that of isoamylbromide remained unchanged. He concluded that, ${ }^{2}$ as proposed by Schallamach, ${ }^{1}$ mixing of these two liquids does not occur at a molecular scale and aggregates of 1-propanol persist in isoamylbromide. The average size of the aggregate in the liquid mixture would be large enough that each molecule in it would show its own dielectric relaxation partly modified by the nature and concentration of the second component. More recently, Murthy and Tyagi ${ }^{3}$ studied another alcoholalkyl halide mixture, namely, 4-methyl-3-heptanol and 1-bromobutane, which also showed two relaxation processes, and attributed the slower process to the $\alpha$ relaxation in the

${ }^{a)}$ Electronic mail: jvij@tcd.ie mixture and the faster process, which occurred, below the calorimetric $T_{g}$ of the mixture, possibly to a $\beta$ relaxation.

The dielectrically or mechanically observed $\alpha$-relaxation process of an ultraviscous liquid is attributed to the ability of molecules to diffuse translationally and rotationally in its structure, which becomes biased by the electrical and mechanical stresses. As a liquid is cooled, its structure changes, and the relaxation time and viscosity increase. When this time becomes equal to $10^{3} \mathrm{~s}$ or the viscosity $\eta$ becomes equal to $\sim 10^{13.3} \mathrm{P}$, the liquid is said to vitrify or become rigid. The temperature at which these two values are reached on cooling is referred to as $T_{g}$. ${ }^{4,5}$ Dielectric and mechanical relaxations of a material are characterized by the relaxation time, the distribution of relaxation times, the equilibrium permittivity, and the limiting high-frequency pemittivity. In the absence of any relaxation, the last of these yields information on the optical and infrared polarizations. Currently, four features of the $\alpha$-relaxation process are regarded as characteristic of liquids; (i) It occurs with an asymmetric distribution of times, ${ }^{5,6}$ which is interpreted in terms of dynamic heterogeneity; ${ }^{7,8}$ (ii) the temperature dependence of its relaxation time follows a Vogel-Fulcher-Tammann type relation, ${ }^{9-11}$ (iii) its dielectric relaxation strength increases on cooling; and (iv) its relaxation time is quantitatively related to the relaxation time of the Johari-Goldstein (JG) process, ${ }^{12,13}$ which is the faster and localized motion whose occurrence leads to the large-scale diffusion involved in the 
$\alpha$ relaxation, ${ }^{14-16}$ and whose equilibrium dielectric properties show features that are characteristic of the $\alpha$-relaxation process. ${ }^{17}$ The dielectric relaxation time of the $\alpha$-relaxation process $\tau$ is regarded as directly proportional to $\eta$ according to Maxwell's relation $\tau=\eta / G_{\infty}$, where $G_{\infty}$ is the instantaneous shear modulus of the liquid. But recent studies have shown that $G_{\infty}$ itself varies with the temperature, as the liquid's structure varies. ${ }^{18,19}$ When relaxation studies are performed on apparently homogeneous mixtures of two liquids, there is an additional difficulty in considering their hydrodynamics in terms of $\eta$ and $G_{\infty}$, because one type of molecules in its ultraviscous liquid may aggregate at submicroscopic scales. Hence, it becomes uncertain as to how $\eta$ and $G_{\infty}$ of either of the two interdispersed liquid phases can be defined at a submicron scale. Moreover, it is expected that interfacial effects have a role in the properties of such incipient phaseseparated liquid mixtures.

Phase separation of a liquid mixture into nanometer-size regions has a consequence for its dielectric and other properties. To gain insight into its effect on the $\alpha$-relaxation behavior, we have initiated a detailed study of such mixtures by dielectric spectroscopy. Here we report a study of a mixture of a long chain alcohol, 5-methyl-2-hexanol, in isoamylbromide, a slightly shorter molecule. We believe that, in addition to their academic value, such studies are important from the point of view of understanding certain biological process as well as in the use of pharmaceuticals in which a hydrogenbonded drug needs to be mixed with dipolar non-hydrogen bonded liquids. We have already reported dielectric spectroscopy studies of both liquids in their pure states. ${ }^{20,21}$ Therefore, a comparison of the dielectric properties of the mixture with those of its (same sample) components, which is needed for our interpretation, is also possible.

\section{EXPERIMENTAL METHODS}

5-methyl-2-hexanol of $99.5 \%$ purity was obtained from Fluka AG. It was a sample from an earlier study. ${ }^{21}$ Isoamylbromide $(96 \%)$ was purchased from Aldrich, and it was also a sample from an earlier study, ${ }^{20}$ which had been redistilled prior to these experiments. Both are low viscosity liquids at ambient temperature. A mixture of composition: $1 \mathrm{~mol}$ 5-methyl-2-hexanol and $4 \mathrm{~mol}$ isoamylbromide was prepared by accurate weighing. It is referred to as a $20 \mathrm{~mol} \%$ 5-methyl-2-hexanol-isoamylbromide mixture. The spectra of the dielectric permittivity and loss, $\varepsilon^{\prime}$ and $\varepsilon^{\prime \prime}$, were measured in real time over the frequency range of $0.4 \mathrm{~Hz}-10 \mathrm{MHz}$ by means of a Novocontrol Alpha-A frequency response analyzer and a ZG4 dielectric interface in two-wire mode. A stainless steel variable multiplate capacitor with gold-plated electrodes and a nominal maximum air capacitance of $27 \mathrm{pF}$ were used. The capacitor and a $\mathrm{Pt} 100 \Omega$ resistance temperature sensor were inserted into a glass vial containing the liquid mixture, ensuring no air bubbles were trapped. The sample was cooled from ambient temperature to liquid $\mathrm{N}_{2}$ temperature. When the $\varepsilon^{\prime}$ and $\varepsilon^{\prime \prime}$ spectra were measured isothermally or, in general, the temperature of the sample was measured at $20 \mathrm{~s}$ intervals with a multimeter connected to the sensor immersed in the sample. The maximum deviation

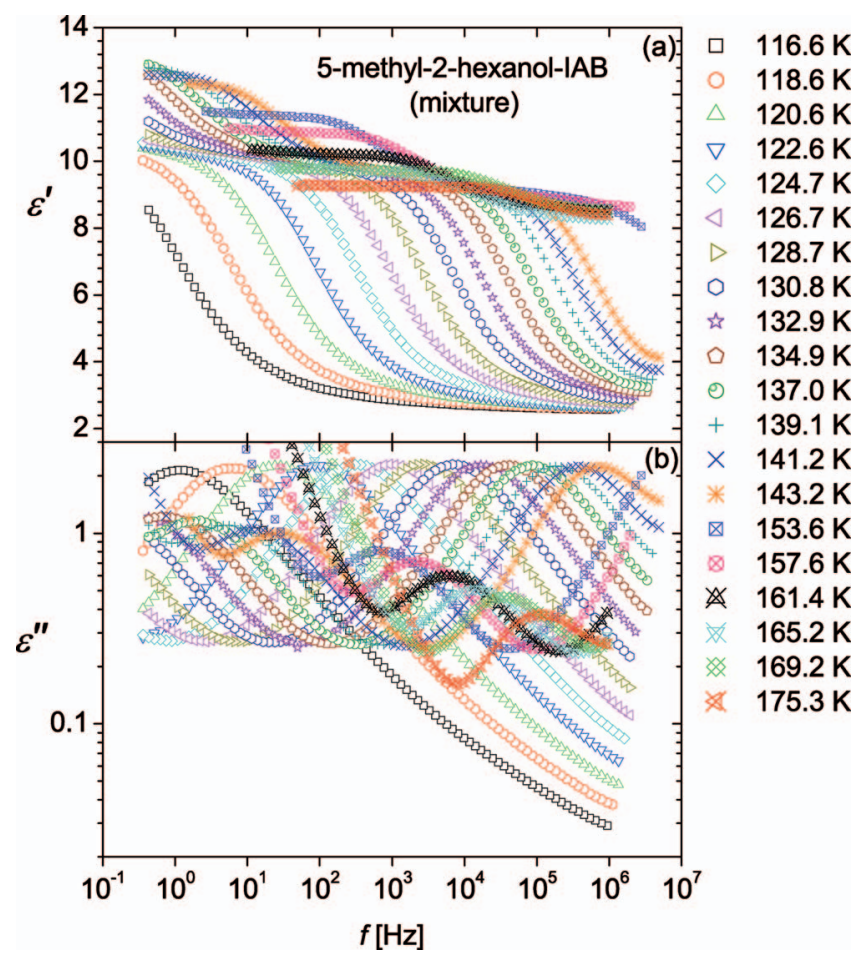

FIG. 1. (Color) The dielectric permittivity $\varepsilon^{\prime}$ (a) and loss $\varepsilon^{\prime \prime}$ (b) spectra of 5-methyl-2-hexanol-isoamylbromide (1:4 molar ratio) mixture. The lowfrequency peak in (b) is due to the relaxation of the 5-methyl-2-hexanol and the high-frequency peak due to that of isoamylbromide.

from the mean temperature was $<0.1 \mathrm{~K}$ after stabilization. The measurement time for the spectra was on the average of $240 \mathrm{~s}$.

\section{RESULTS}

Several $\varepsilon^{\prime}$ and $\varepsilon^{\prime \prime}$ spectra of the 5-methyl-2-hexanolisoamylbromide mixture were obtained over the temperature range of 101-181.5 K. Typical spectra at the temperatures when the dispersion and absorption features were clearly evident are shown in Figs. 1(a) and 1(b). Over most of the temperature range of this study, the spectra show two dispersion regions in $\varepsilon^{\prime}$ and two peaks corresponding to these dispersions in $\varepsilon^{\prime \prime}$. As mentioned earlier here, Schallamach ${ }^{1}$ had reported that the plots of fixed frequency values of $\varepsilon^{\prime}$ and $\varepsilon^{\prime \prime}$ against the temperature of 1-propanol isoamylbromide and geraniol-isoamylbromide mixtures had shown two steps in $\varepsilon^{\prime}$ and two peaks in $\varepsilon^{\prime \prime}$. A later study of the relaxation spectra of 1-propanol isoamylbromide mixture by Denney ${ }^{2}$ had shown two relaxation regions in the Cole-Cole plots of $\varepsilon^{\prime \prime}$ against $\varepsilon^{\prime}$, the lowest frequency arc in the plots corresponding to the relaxation of 1-propanol and the high-frequency arc corresponding to that of isoamylbromide. The dielectric relaxation time of isoamylbromide is shorter than that of 5-methyl-2hexanol at the same temperature. ${ }^{20,21}$ Therefore, at higher temperatures, $153.6-175.3 \mathrm{~K}$ in Fig. 1, the $\varepsilon^{\prime}$ dispersion and $\varepsilon^{\prime \prime}$ peak in the mixture are due to the Brownian motions of 5-methyl-2-hexanol in the mixture. The $\varepsilon^{\prime}$ dispersion and $\varepsilon^{\prime \prime}$ peak at the lowest temperatures, 116.6-132.9 K, in Fig. 1 are due to the motions of isoamylbromide. At the lowest temperature at which the isoamylbromide $\varepsilon^{\prime}$ dispersion and $\varepsilon^{\prime \prime}$ peak appear in the frequency window of the experiment 


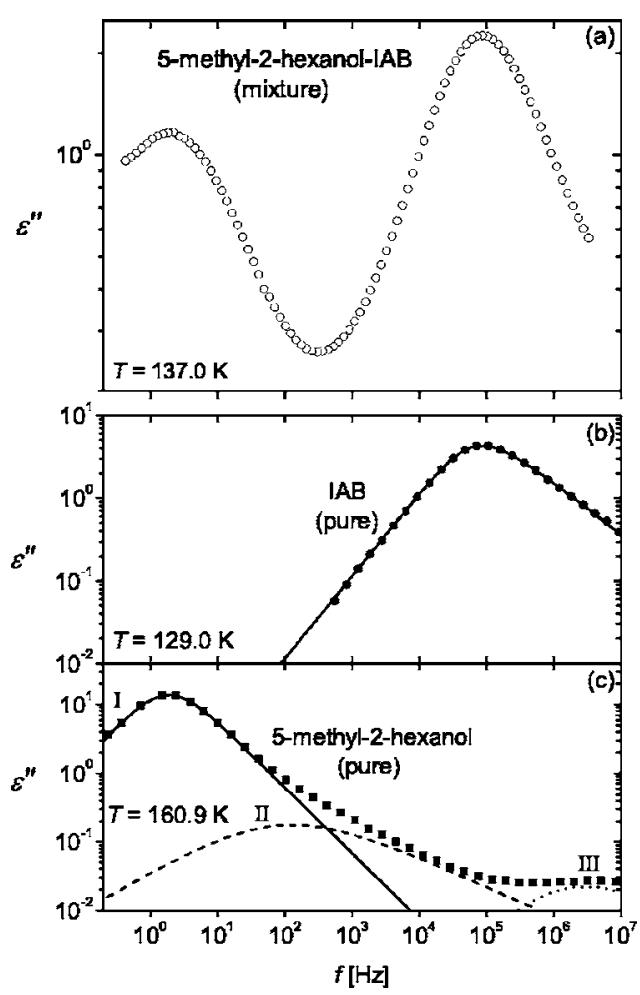

FIG. 2. (a) The dielectric loss spectra of 5-methyl-2-hexanolisoamylbromide mixture at $137.0 \mathrm{~K}$. (b) The corresponding spectra for pure isoamylbromide at $129.0 \mathrm{~K}$. (c) The corresponding spectra for 5-methyl-2hexanol at $160.9 \mathrm{~K}$. Note the partial overlap of processes II and III of pure 5-methyl-2-hexanol with the spectra of pure isoamylbromide. This would also lead to slight overlap in the same spectral region of the mixture, which prevents an appropriate analysis of processes II and III of 5-methyl-2hexanol in the mixture and complete characterization of the contribution of each substance to the mixture spectrum.

$(116.6 \mathrm{~K})$, the relaxation time of isoamlybromide molecules estimated from the frequency of maximum loss would be $\sim 0.14 \mathrm{~s}$. At this temperature, the 5-methyl-2-hexanol has, in fact, vitrified $\left(T_{g} \sim 121.4 \mathrm{~K}\right)$, and the relaxation time of 5-methyl-2-hexanol in the mixture is thus many orders of magnitude longer than that of the isoamylbromide. The Brownian diffusion of isoamylbromide still occurs when 5-methyl-2-hexanol is in the glassy state. The $\varepsilon^{\prime}$ dispersion and $\varepsilon^{\prime \prime}$ peak of 5-methyl-2-hexanol would still be observable but in a much lower frequency range inaccessible by this experimental technique.

An earlier study ${ }^{21}$ has shown that 5-methyl-2-hexanol has three relaxation processes, one of which appears as a Debye peak (i.e., an exponential decay of polarization or single relaxation time) in the ultraviscous state and the other two are resolved from the high-frequency $\varepsilon^{\prime}$ and $\varepsilon^{\prime \prime}$ data and show an asymmetric distribution of relaxation times. In contrast isoamylbromide had only two relaxation processes, one of which appeared as an $\varepsilon^{\prime}$ dispersion and $\varepsilon^{\prime \prime}$ peak and the second process was resolved from the high-frequency part of the spectra. Both processes showed an asymmetric distribution of relaxation times. ${ }^{20}$ The fastest relaxation in both isoamylbromide and 5-methyl-2-hexanol was attributed to the JG relaxation. ${ }^{20,21}$ To show their spectral positions and features relative to the spectra of the mixture, we have plotted the $\varepsilon^{\prime \prime}$ spectrum of the mixture at $137.0 \mathrm{~K}$ in Fig. 2(a) and spectra of 5-methyl-2-hexanol and of isoamylbromide in Figs. 2(b) and 2(c). The temperature of $137.0 \mathrm{~K}$ was chosen for this plot because it is the lowest temperature at which the $\varepsilon^{\prime \prime}$ peaks for both substances are seen together within the frequency window of the experiment. Note that the temperatures for comparison of the pure liquids $(129.0 \mathrm{~K}$ for pure isoamylbromide and $160.9 \mathrm{~K}$ for pure 5-methyl-2-hexanol) are different, both from each other and the temperature at which the mixture spectrum was measured. Data were not available for pure isoamylbromide at $137.0 \mathrm{~K}$ because the pure compound began to crystallize at $T$ above $\sim 129 \mathrm{~K} .{ }^{20}$ At $137 \mathrm{~K}$, pure 5-methyl-2-hexanol is well below its $T_{g}$, deeply in the glassy state, and the relaxation time of its $\alpha$ process cannot be determined. For that reason, the $\varepsilon^{\prime \prime}$ spectrum of the mixture measured at $160.9 \mathrm{~K}$, where pure 5-methyl-2hexanol's relaxation time is close to that of the mixture at $137 \mathrm{~K}$, was chosen for comparison. In Figs. 1(a) and 1(b), it is evident that some of the spectral features of the $\alpha$ relaxation and the JG relaxation overlap the spectra measured here, and as a result it is difficult to unambiguously resolve the $\varepsilon^{\prime}$ and $\varepsilon^{\prime \prime}$ spectra of the mixture into a total of five relaxation regions and dc conductivity. Because of that difficulty, we proceed by assuming that the high-frequency relaxation processes, i.e., processes II and III of 5-methyl-2-hexanol, ${ }^{21}$ do not significantly affect the $\varepsilon^{\prime \prime}$ peak position of isoamylbromide. And, therefore, instead of analyzing the spectra of the two liquids in the mixture into their respective high-frequency components, we have determined the $\varepsilon^{\prime \prime}$ peak frequency $f_{m}$, from the spectra shown in Fig. 1 (and from a number of other spectra excluded from Fig. 1) by fitting a high order polynomial (up to order 7) to the points around $f_{m}$, and calculated the dielectric relaxation time from the relation $\tau=1 / 2 \pi f_{m}$.

Figure 3 shows the plots of $\log _{10} \tau$ against the temperature for the relaxation due to molecular motions in 5-methyl2-hexanol and that for the relaxation of molecules in isoamylbromide. For comparison, the plots of $\tau$ of pure 5-methyl-2-hexanol taken from Ref. 21 and of isoamylbromide taken from Ref. 20 are also shown. The Vogel-FulcherTammann equation, ${ }^{9-11} \log \tau=-13.89+[825.8 /(T-72.5)]$, fits the data for 5-methyl-2-hexanol in the isoamylbromide mixture. From the earlier study of pure 5-methyl-2-hexanol, ${ }^{21} \log \tau=-14.43+[1205 /(T-79.8)]$ For isoamylbromide, $\tau$ values in the mixture and in the pure state are similar and are fitted reasonably well by the same relation as given before, ${ }^{20} \log \tau=-17.61+[787 /(T-68.5)]$ as seen by the line through the data in Fig. 3. It should be stressed that these parameters do not imply that only a single VogelFulcher-Tammann equation would fit the data up to a formally infinite temperature where $\tau$ is assumed to be in the range of $10^{-14}-10^{-12} \mathrm{~s}$.

To determine whether there is a change in the shape of the relaxation spectra of the mixture's components, we have shown the Cole-Cole ${ }^{22}$ plots of the mixture in Fig. 4 at 118.6, 141.2 , and $153.6 \mathrm{~K}$. These temperatures were chosen such as to reveal the distinction between the Cole-Cole arc, arising from mainly isoamylbromide at $118.6 \mathrm{~K}$, from both 5-methyl-2-hexanol and isoamylbromide at $141.2 \mathrm{~K}$, and mainly from 5-methyl-2-hexanol at $153.6 \mathrm{~K}$. Of the two arcs 


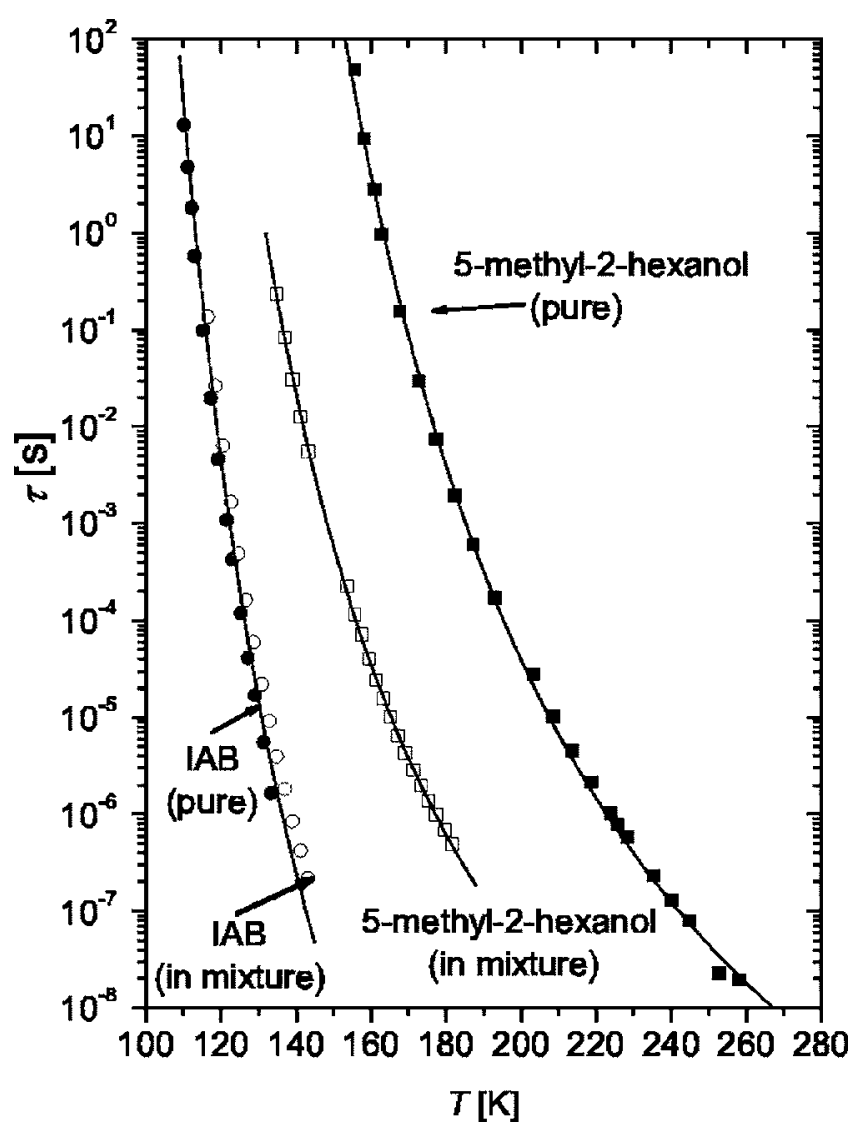

FIG. 3. The relaxation time obtained from the frequency of maximum loss of 5-methyl-2-hexanol-isoamylbromide mixture is plotted against the temperature. For comparison, the data for pure 5-methyl-2-hexanol (filled squares) and pure isoamylbromide (filled circles) are also plotted. The continuous lines are the fits to the equation $\log _{10} \tau=A+\left[B /\left(T-T_{0}\right)\right]$, where $A$ $=-17.61, B=787 \mathrm{~K}$, and $T_{0}=68.5 \mathrm{~K}$ for pure isoamylbromide (Ref. 20) and the isoamylbromide component of the mixture. For pure 5-methyl-2-hexanol (Ref. 21) $A=-14.43, B=1205 \mathrm{~K}$, and $T_{0}=79.8 \mathrm{~K}$, and for 5-methyl-2hexanol in the mixture, $A=-13.89, B=825.8 \mathrm{~K}$, and $T_{0}=72.5 \mathrm{~K}$.

seen at $141.2 \mathrm{~K}$ in Fig. 4, the one on the right hand side in the low-frequency range is due to the $\alpha$ relaxation of 5-methyl-2-hexanol in the mixture. It is clearly more skewed at the high frequency end. In this respect it remarkably differs from the spectra for pure 5-methyl-2-hexanol which had shown a semicircular arc characteristic of the Debye-type relaxation that had relaxed $\sim 97 \%$ of its total orientation polarization. The arc on the left hand side at high frequencies is due to the reorientational motion of isoamylbromide. It shows an asymmetric distribution of relaxation times of the Davidson-Cole ${ }^{23}$ type.

An extrapolation for $\tau$ of 5-methyl-2-hexanol in the mixture from the fitted equation, $\log \tau=-13.89+[825.8 /(T$ $-72.5)]$ in Fig. 3, yields a value of $3.1 \mathrm{ks}$ at $120 \mathrm{~K}$ which is $3 \times 10^{5}$ times the isoamylbromide relaxation time in the mixture at the same temperature [estimated by interpolation with its own Vogel-Fulcher-Tammann equation, $\log \tau=-15.44$ $+[591.4 /(T-75.9)]$, rather than the one for the fit to the pure isoamylbromide from Ref. 20]. This means that the structure of the (incipient) phase-separated 5-methyl-2-hexanol is kinetically frozen at $T \leqslant 121 \mathrm{~K}$, and the isoamylbromide molecules in the remainder of the ultraviscous phase diffuse in the regions surrounding the glassy phase of 5-methyl-2hexanol.

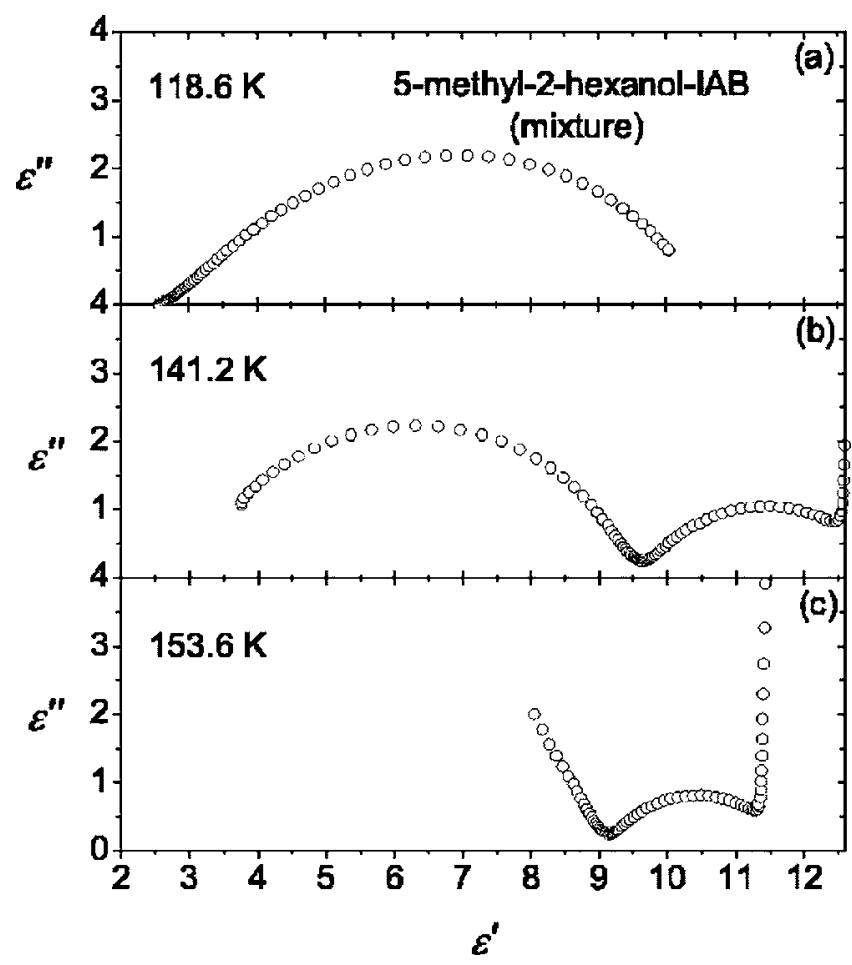

FIG. 4. The complex plane plots of the dielectric loss and permittivity of the 5-methyl-2-hexanol-isoamylbromide mixture at three temperatures: (a) $118.6 \mathrm{~K}$, (b) $141.2 \mathrm{~K}$, and (c) $153.6 \mathrm{~K}$.

The dielectric relaxation strength $\Delta \varepsilon$ of pure 5-methyl2-hexanol spectra had been resolved into three relaxation processes, named I, II, and III, of which I is the Debye-single relaxation time which carries $\sim 97 \%$ of the polarization, and process III, which carries about $0.3 \%$, is suggested to be due to the JG relaxation. (It should be noted that a Debye relaxation has now been observed in the ultraviscous state of a dilute solution of di-n-butyl ether, a non-hydrogen-bonded liquid, in 3-methylpentane, and its total relaxation spectra has been also resolved into three relaxation regions, ${ }^{24}$ as for other hydrogen-bonded liquids. ${ }^{21}$ This shows that hydrogen bonding in a liquid is unnecessary for a Debye-relaxation process.) As shown in Fig. 2 and discussed here, there would be a considerable ambiguity in resolving the relaxation spectra of 5-methyl-2-hexanol in the mixture into three relaxation regions. Even if a shift of the spectra for pure 5-methyl-2hexanol to higher frequencies in its isoamylbromide mixture, to be discussed here later, is taken into account, relaxations II and III of the 5-methyl-2-hexanol spectra would overlap part of the spectra of isoamylbromide. Because of the consequent ambiguity in the analysis, we do not analyze the spectral regions of 5-methyl-2-hexanol in the mixture into its components I, II, and III to determine $\Delta \varepsilon$, the orientational polarization contribution to the susceptibility, for 5-methyl-2hexanol. Instead, we use the midpoint value of $\varepsilon^{\prime}$ as an estimate for the sum of the high-frequency permittivity of the mixture $\varepsilon_{\infty}$ and the orientational polarization contribution of the isoamylbromide component to the mixture permittivity. Any remaining contribution to $\varepsilon_{s}$ is then taken to be $\Delta \varepsilon$ of the 5-methyl-2-hexanol component. $\varepsilon_{\infty}$ was estimated from a spectrum measured at a low temperature where the contribution to the permittivity from relaxation processes is minimal 


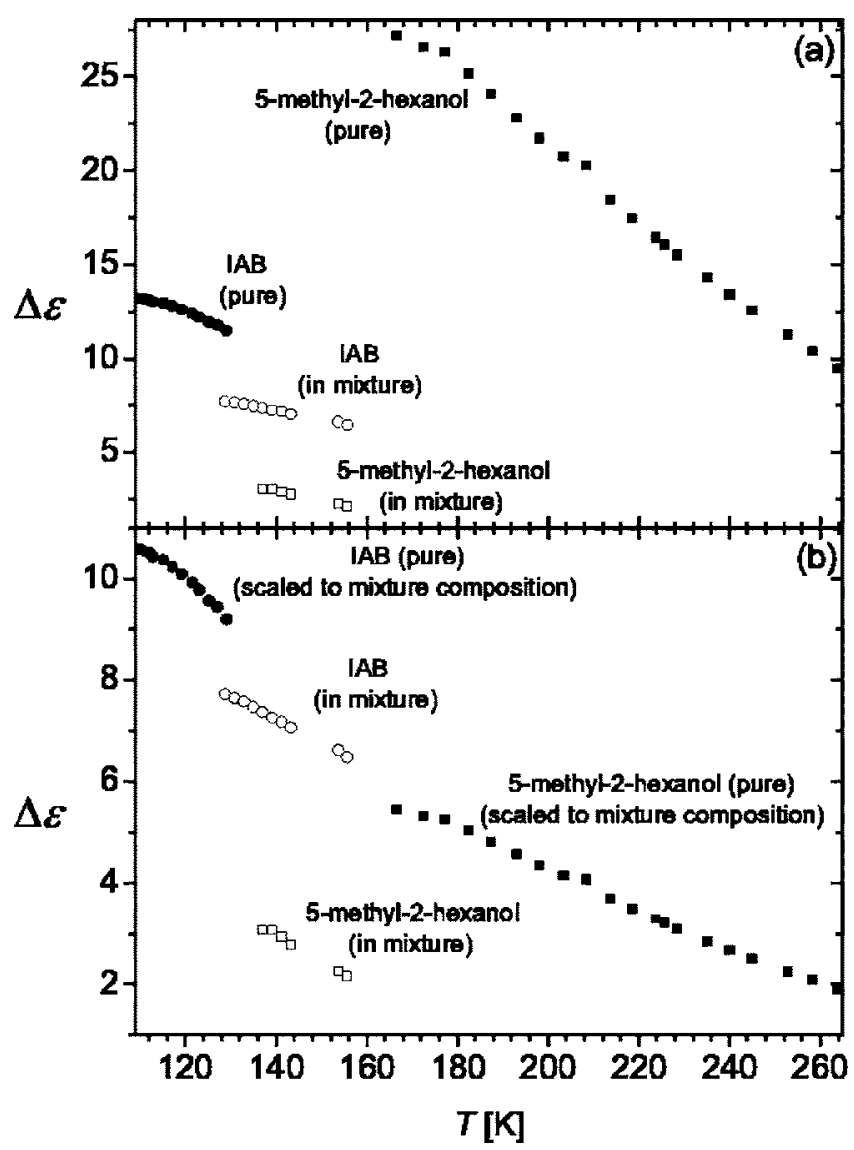

FIG. 5. (a) The contribution to the dielectric permittivity of the mixture from the orientational polarization of the 5-methyl-2-hexanol and isoamylbromide components plotted as a function of temperature. The unscaled values measured for pure 5-methyl-2-hexanol and isoamylbromide are shown for comparison. (b) The expected contribution to the dielectric permittivity of the mixture from the orientational polarization of pure 5-methyl2-hexanol and pure isoamylbromide is plotted against the temperature. This is shown by scaling the values for the pure substances to the mole fraction of each present in the mixture. The actual values measured for 5-methyl-2hexanol and isoamylbromide in the mixture are included for comparison.

$(102.9 \mathrm{~K})$. Knowledge of $\varepsilon_{\infty}$ then allows $\Delta \varepsilon$ of isoamylbromide in the mixture to be estimated also. These values are plotted against the temperature in Fig. 5. For comparison, $\Delta \varepsilon$ values of pure 5-methyl-2-hexanol and pure isoamylbromide are also plotted in Fig. 5.

To gain an approximate idea of the distribution of relaxation times, the spectra were analyzed by fitting the sum of a Davidson-Cole ${ }^{23}$ (with an asymmetric broadening parameter $\beta$ ) and a Cole-Cole ${ }^{22}$ relaxation term (the latter included to represent any JG relaxation contribution at the lower temperatures) to the isoamylbromide $\varepsilon^{\prime \prime}$ peak for each temperature in which it occurred within the measurement frequency window. The data on the low-frequency side of the peak were excluded as these should contain the most influence from the higher frequency relaxations of the 5-methyl-2hexanol component. This contribution should be largest at lower temperatures because the relaxation times of the isoamylbromide and 5-methyl-2-hexanol in the mixture become closer as the temperature is decreased and the strength of process II increases (see Fig. 3 and 4). In order to obtain as many points as possible beyond the peak in the frequency window for a reliable fit while minimizing the influence of the 5-methyl-2-hexanol relaxation processes, we use the spectra for the mixture at $124.7 \mathrm{~K}$ with the isoamylbromide $\varepsilon^{\prime \prime}$ peak near the center of the frequency window. It yields $\beta=0.52$ for isoamylbromide in the mixture. For pure isoamylbromide, $\beta=0.61$ at $125.2 \mathrm{~K}^{20}$

\section{DISCUSSION}

\section{A. The relaxation time}

The viscosity of pure 5-methyl-2-hexanol is higher than that of isoamylbromide at ambient temperature, and its $\tau$ is at least $10^{8}$ times the value for isoamylbromide at a temperature near $120 \mathrm{~K}$. It is therefore expected that not only their homogeneous mixture would show just one relaxation process, but also that the viscosity of the mixture would be higher than that of pure isoamylbromide. Hence, hydrodynamically speaking, $\tau$ of isoamylbromide in the mixture at $120 \mathrm{~K}$ would be at least an order of magnitude higher than in the pure state. Instead, $\tau$ of isoamylbromide in the mixture in Fig. 3 is within the experimental and analytical uncertainties of its value in the pure state. This shows that the isoamylbromide motions are not affected by the presence of 5-methyl-2-hexanol, i.e., isoamylbromide phase separates from 5-methyl-2-hexanol molecules in the mixture. Alternatively stated, 5-methyl-2-hexanol aggregate to form clusters, leaving the isoamylbromide in a relatively pure state in the mixture. The refractive index of 5-methyl-2-hexanol is 1.418 and that of isoamylbromide is 1.442 at ambient temperature. ${ }^{25}$ According to the Fresnel equation, this difference is sufficient to produce a considerable optical contrast in the mixture to make it somewhat opaque. But the mixture at ambient temperature is found to be an optically transparent solution. Since dielectric spectra show two different regions of relaxation, the optical transparency indicates that phase separation does not occur into 5-methyl-2-hexanol aggregates or particles larger than the wavelength of light, that is, it must occur at a submicrometer or a nanometer scale.

There is a further indication that the mixture is not homogeneous at a submicrometer scale. This comes from the generally noted fact that in a molecularly homogeneous solution $\tau$ of the low-viscosity component increases in the mixture and that of the high-viscosity component decreases, i.e., the $\tau$ values of the two move in opposite directions. This has been recently confirmed from detailed studies of solutions of alcohols in nonpolar solvents ${ }^{3}$ and solutions of some alcohols in slightly polar solvents. ${ }^{26}$ To elaborate, Murthy and Tyagi $^{3}$ have found that $\tau$ of 4-methyl-3-heptanol decreases in its mixture with 1-bromobutane (Fig. 11, Ref. 3), but they did not ascertain whether the faster relaxation they had observed in the mixture was a $\beta$-relaxation process or was it due to relaxation of 1-bromobutane molecules in the mixture. In the present study, $\tau$ of 5-methyl-2-hexanol in the mixture decreases but $\tau$ of the isoamylbromide does not change. There is also a JG relaxation process from the motions of isoamylbromide molecules in the mixture, as had been observed in its pure state, ${ }^{20}$ but this cannot be separately analyzed due to the interference of the alcohol components' relaxations in the mixture. 
The relaxation time of 5-methyl-2-hexanol in the phaseseparated mixture at $180 \mathrm{~K}$ is a factor of $1.6 \times 10^{7}$ less than that in the pure state at $160 \mathrm{~K}$. In the pure state it forms intermolecular hydrogen bonds such as to produce a linear chain structure. This interpretation is largely based on the interpretation of the unusually high $\varepsilon_{s}$ value for water, ${ }^{27}$ in which similar intermolecular hydrogen bonds have been observed by a variety of techniques, and in which such bonds cause a (dynamic) short range dipolar correlation that raises the dipole moment that reorients when a molecule reorients. We suggest that because of the large size of the molecule itself and hindrance to the $-\mathrm{OH}$ group there would be much fewer 5-methyl-2-hexanol molecules in its nanometer-size cluster than in a micrometer-size cluster, i.e., the extent of hydrogen bonding in the clusters would be significantly less. The presumably spherical shape of the cluster needed to minimize its surface energy would further constrain the alignment of $-\mathrm{OH}$ groups necessary for the hydrogen bonding. Since each molecule forms two hydrogen bonds, one as a donor of a proton and another as an acceptor, the number of hydrogen bonds that need to break before a reference dipole and its dipolar environment that can orient would be less than two and $\tau$ hence would decrease. We propose that the $10^{5}$-fold decrease in $\tau$ of 5-methyl-2-hexanol in the mixture reflects a decrease in the number of intermolecular hydrogen bonds, mainly due to the small size of the cluster and the steric hindrance to the formation of hydrogen bonds by the (seven carbon atoms) hydrocarbon chain containing the $\mathrm{OH}$ group.

It should be noted that Denney ${ }^{3}$ had found that $\tau$ of 1-propanol had increased when it was mixed with isoamylbromide in the same composition of 1:4 mole as 5-methyl2-hexanol is mixed in our study. This is opposite to the decrease observed here. (Note that viscosity measurements on binary mixtures of other monohydroxy alcohols have shown that it monotonically decreases as 1-bromobutane is added to an alkanol, ${ }^{28}$ but these data do not refer to the 1-propanol, isoamylbromide or the mixture studied here and it is not known how the viscosity would change on mixing.) It is conceivable that for the relatively small molecule of 1-propanol a denser cluster forms as a result of hydrogen bonding, which increases its $\tau$ in the mixture.

It has been found previously that the relaxation time of the H-bonded liquid propylene glycol contained in mesopores $(7.5-2.5 \mathrm{~nm})$ of a sol-gel glass matrix may either decrease or increase depending on whether the pores are treated with hexamethyldisilazane (which silanizes - $\mathrm{OH}$ groups on the pore walls) or not. ${ }^{29,30}$ In the untreated pores there are strong H-bonding interactions between the pore walls and the propylene glycol molecules at the interface, which increases their relaxation time. When the pores are treated in specific ways, these interactions are reduced and the relaxation then becomes slightly faster than in the bulk. A more recent differential scanning calorimetry study ${ }^{31}$ has shown that $T_{g}$ of another H-bonded liquid, glycerol, confined to hexagonal mesoporous silica with pore sizes of 26.4 $-2.6 \mathrm{~nm}$ (untreated to remove $-\mathrm{OH}$ bonds) increases with decreasing pore size as a result of increasing importance of these interface effects, while $T_{g}$ of a non- $\mathrm{H}$ bonded liquid (o-terphenyl) is reduced by $\sim 20 \mathrm{~K}$ for the smallest pore size because of the effect of the confinement on the growth of cooperatively rearranging regions in the supercooled liquid with a decrease in the temperature. Such experiments suggest that the interaction between the alcohol molecules on the interface of the clusters with the larger component and perhaps the effect of confinement on $T_{g}$ in such nanosize clusters could also be factors in determining whether $\tau$ of the alcohol increases in the mixture or not.

\section{B. The dielectric relaxation strength and the distribution parameter}

The plots in Fig. 5(a) show that the dielectric relaxation strength $\Delta \varepsilon$ of both 5-methyl-2-hexanol and isoamylbromide in the solution are lower than in their respective pure states. This is expected because their amounts are only a fraction of a mole in the mixture. Were the solution homogeneous, the $\Delta \varepsilon$ value of each would be obtained by scaling the pure state $\Delta \varepsilon$ values by their relative mole fractions and the square of the effective dipole moments. Therefore, to investigate if there are additional dipolar effects in the solution that alter the $\Delta \varepsilon$ values determined here, we scale the $\Delta \varepsilon$ of pure 5-methyl-2-hexanol and pure isoamylbromide by their corresponding mole fraction in the mixture, i.e., multiply $\Delta \varepsilon$ for pure 5-methyl-2-hexanol by its mole fraction 0.2 and $\Delta \varepsilon$ of isoamylbromide by its mole fraction 0.8 , and assume that the effective dipole moment does not change. These scaled values are plotted in Fig. 5(b). They show that the measured $\Delta \varepsilon$ of 5-methyl-2-hexanol in the mixture [open squares in Fig. $5(\mathrm{~b})$ ] is about $36 \%$ of the scaled $\Delta \varepsilon$ value at $155.7 \mathrm{~K}$ [filled squares in Fig. 5(b)]. Similarly, the measured $\Delta \varepsilon$ of isoamylbromide at $130.0 \mathrm{~K}$ [circles in Fig. 5(b)] is about $83 \%$ of the scaled value [filled circles in Fig. 5(b)] at $130 \mathrm{~K}$.

In the statistical mechanical theory of dielectrics the lower values observed for isoamylbromide may indicate a slightly decreased density of isoamylbromide regions in the phase separated state in the mixture and/or a slight decrease in their short range dipolar orientational correlation. In contrast, the threefold lower value of $\Delta \varepsilon$ for 5-methyl-2-hexanol indicates a decrease in its density in the clusters but it indicates mostly a decrease in the dipolar orientational correlation from its value in the pure state to that in the phaseseparated or clustered state in the mixture. Dielectric polarization in hydrogen-bonded and strongly interacting dipolar liquids has been interpreted in terms of a statistical theory of dipolar orientational correlation given by Onsager, ${ }^{32}$ Kirkwood, ${ }^{33}$ and Fröhlich ${ }^{34}$ in terms of a quantity $g$, which alters the effective dipole moment from its vapor phase value. If the effective dipole moment of 5-methyl-2hexanol remained unchanged, then its scaled $\Delta \varepsilon$ would be close to the measured value. The threefold decrease from the scaled $\Delta \varepsilon$ for pure 5-methyl-2-hexanol in the mixture to the actual value, therefore, reflects a significant decrease in $g$. This would be expected if constraints to intermolecular hydrogen bonding became prominent in the phase-separated state or else some of the molecules hydrogen-bonded in an antiparallel manner into dimers or multimers such that the net effective dipole moment decreased. ${ }^{35-38}$ It should also be 


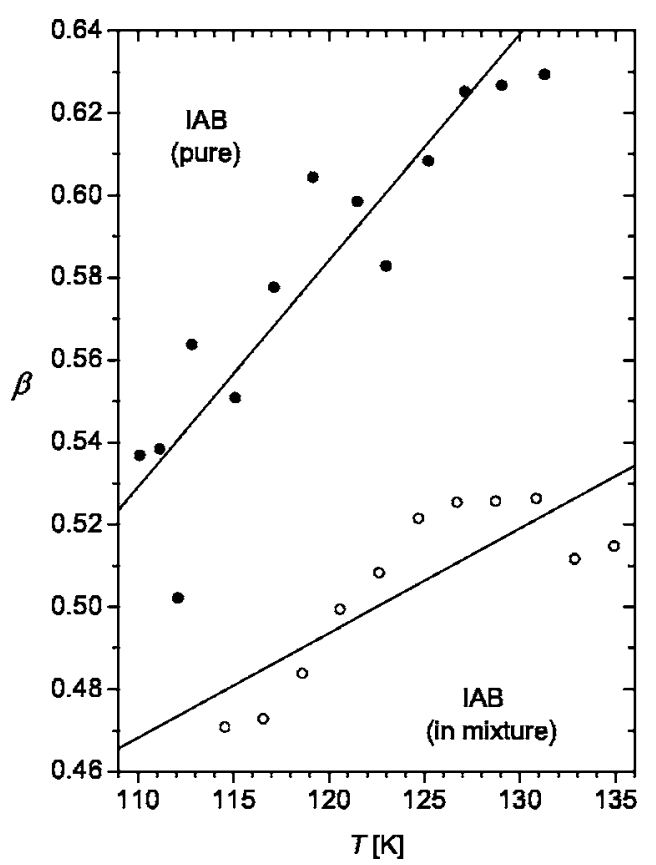

FIG. 6. The asymmetric distribution parameter $\beta$ for isoamylbromide in the mixture and in the pure state is plotted against the temperature.

noted that the product $T \Delta \varepsilon$ for pure 5-methyl-2-hexanol varies from 4666 at $177.3 \mathrm{~K}$ to 2498 at $263.7 \mathrm{~K}$.

We now discuss the shape of the relaxation spectra. For the Debye relaxation in pure 5-methyl-2-hexanol, $\beta=1$. But as is evident from the skewness in the high-frequency part of the right hand arc in the Cole-Cole plots at 141.2 and 153.6 K shown in Fig. 4, it is less in the mixture. As mentioned earlier here, the high-frequency relaxation processes of 5-methyl-2-hexanol partly overlap the $\alpha$ relaxation from isoamylbromide and this makes any analysis for its $\beta$ parameter uncertain. Nevertheless, as stated previously, $\beta$ of isoamylbromide can be determined by taking the $\varepsilon^{\prime \prime}$ points on the high-frequency side of $f_{m}$ for isoamylbromide and assuming a Davidson-Cole distribution for the $\alpha$-relaxation contribution to $\varepsilon^{\prime \prime}$ and a Cole-Cole one for any remaining JG-relaxation contribution to $\varepsilon^{\prime \prime}$. Its value is plotted against the temperature in Fig. 6 . For both the pure liquid and the mixture $\beta$ decreases with the decrease in the temperature, as expected. The $\beta$ value of $\sim 0.52$ at $124.7 \mathrm{~K}$ for the mixture is significantly less than the value of 0.61 for the pure isoamylbromide at $125.2 \mathrm{~K}$.

Evidently, the presence of 5-methyl-2-hexanol aggregates increases the distribution of relaxation times of isoamylbromide in the mixture without significantly altering its relaxation time. In the dynamic heterogeneity conjecture $^{7,8}$ this would mean that the presence of nanoscopic heterogeneity of a different constituent adds to the dynamic heterogeneity of isoamylbromide regions. We conclude that the distribution of the relaxation time parameter decreases for both 5-methyl-2-hexanol and isoamylbromide when the two are mixed.

\section{Structure of the liquid and the relaxation processes}

It has been argued that the Debye relaxation in alcohols and amides may not be associated with the structural relax- ation or the $\alpha$-relaxation process. ${ }^{39,40}$ There has been experimental evidence from other studies ${ }^{21,41-43}$ that indicate the lack of merit in the arguments presented. ${ }^{41,42}$ Moreover, dielectric relaxation of a variety of unbranched monohydric alcohols has also been studied in $n$-alkane solutions at $298 \mathrm{~K}$ and $1 \mathrm{MHz}-18 \mathrm{GHz}$ frequency range by Schwerdtfeger, et $a l .{ }^{44}$ and the observed Debye relaxation time has been discussed in terms of the usual wait-and-switch model of relaxation in which hydrogen bonds break and then reform. This is the same model for relaxation as for water at high temperatures in which hydrogen-bond fluctuation is attributed to the Debye relaxation or a very small deviation from it towards a symmetric distribution of relaxation times. It involves relaxation of a molecule's environment after breaking of its hydrogen bond, such that it suitably disposes the molecule in order to form a hydrogen bond with another of its molecular neighbor. In relation to this study, it is also difficult to see how this mechanism can be reconciled when molecular clusters of alcohols form in another dipolar liquid. In recent studies, ${ }^{45}$ the Debye-Stokes-Einstein relation of the inverse proportionality between the dc conductivity and dielectric relaxation time has been variously tested and its modification has been suggested. ${ }^{46}$ For the mixture studied here, it is also difficult to determine which of the two relaxation processes, that of 5-methyl-2-hexanol or that of the isoamylbromide, should be related to the dc conductivity in the Debye-Stokes-Einstein equation.

In the recent concern on the nature of the Debye relaxation, discussion of experimental studies has been limited to alcohols and two secondary amides. ${ }^{47}$ There are two underlying reasons for the issues raised in this discussion: Firstly, the Debye relaxation for which $\beta=1$ cannot be reconciled with the correlation between $m$, the steepness of the Oldekop plots $^{48,49}$ at $T=T_{g}$, and $\beta$, because the measured $m$ value is too high for $\beta=1$. Secondly, it is an apparent requirement of the dynamic heterogeneity view of a liquid's structure that $\beta$ be less than 1 . Since the observation of $\beta=1$ cannot be reconciled with these presumptions, it has been proposed that the Debye relaxation in the liquid should be explained by suggesting that it does not refer to Brownian motions or structural fluctuations that lead to a viscous flow in monohydroxy alcohols, even though the Debye process relaxes $\sim 97 \%$ of the polarization. Instead, process II, which shows a distribution of relaxation times and relaxes under $3 \%$ of the orientational polarization, would be related to the viscous flow. As a rationale, it has been suggested that the Debye relaxation corresponding to Process 1 could be related to process II in an unspecified manner, but it has no direct role in viscous flow. ${ }^{26}$

Recently a non-hydrogen-bonded liquid, di- $n$-butyl ether, has been reported to show a Debye-type $\alpha$ relaxation in the ultraviscous state of its $1 \mathrm{wt} \%$ solution in 3-methylpentane. ${ }^{24}$ Its $\tau$ also shows a Vogel-FulcherTammann-type temperature dependence over a wide temperature range down to a temperature approaching $T_{g}$. This also seems to be inconsistent with the connection between $m$ and $\beta$, which is equal to 1 and the abovementioned view on the dynamic heterogeneity in an ultravsicous liquid's structure. Nevertheless, the data for di- $n$-butyl ether were inter- 
preted in terms of the Anderson-Ullman ${ }^{50}$ model for environmental fluctuation and the shape of the relaxation spectra in as much as the orientation of di- $n$-butyl ether dipole occurred in the environment of a faster relaxing 3-methylpentane. This is the same mechanism as was used for interpreting the relaxation in monohydroxy alcohols. ${ }^{51}$ Evidently, hydrogen bonding is unnecessary for the observation of a Debye relaxation in an ultraviscous liquid.

In relation to our study, it is clear that hydrogen bonding does not necessarily produce a Debye relaxation in some alcohols, and there seems to be no a priori need for attributing the Debye relaxation to micelles or other structure in pure monohydroxy alcohols. Some of the long chain alcohols, such as a variety of isomeric octanols which are expected to show a greater ability to form micelles, have indeed shown a distribution of relaxation times, ${ }^{36}$ and di$n$-butyl ether, without hydrogen bonds, has shown a Debye relaxation. ${ }^{24}$ It may seem tempting to suggest the possibility of micelle formation by agglomeration of the hydrogenbonded molecules, and then the possibility of difference between the proton conduction within the micelle and in the bulk. This may give rise to an interfacial polarization at the micelle-liquid interface, but there has been no evidence for such micelles from light scattering techniques, and one expects that their increase in an isoamylbromide mixture would produce a larger Debye relaxation contribution than in the pure state. Studies have shown that a mismatch in the molecular size decreases micelle stability and the foaming property of a liquid mixture. ${ }^{52}$ Our study has shown that the spectra instead becomes broader than the Debye spectra and the contribution to the polarization in the mixture is less than the expected value.

\section{CONCLUSION}

The dielectric relaxation spectra of a long chain alcohol 5-methyl-2-hexanol mixed with isoamylbromide ( $1: 4 \mathrm{~mol} / \mathrm{mol}$ ratio) shows two relaxation regions attributable to the reorientation of the monohydroxy alcohol and of isoamylbromide molecules at different rates. This indicates that 5-methyl-2-hexanol phase separates most likely into nanometer-size regions. The dynamics of 5-methyl-2hexanol in the mixture becomes faster by over five orders of magnitude at $155.7 \mathrm{~K}$, but that of isoamylbromide increases marginally. The permittivities due to orientational polarization in 5-methyl-2-hexanol decreases by a factor of 13.8 from the pure to the mixed state and that due to isoamylbromide decreases by about a factor of 1.5 , when scaling for the mole fraction of each component, are not taken into account. For 5-methyl-2-hexanol especially, the value of the contribution expected when scaling from the pure state to the mixture composition is included and remains much higher than was actually measured in the mixture, indicating a considerable decrease in the orientational polarization as a result of the change in the density and/or short range dipolar orientation. The distribution of relaxation time for isoamylbromide increases in the mixture, indicating a greater dynamic heterogeneity in the presence of aggregates of phase-separated 5-methyl-2-hexanol. The results show that phase separation may lead to a decrease in the relaxation time as the extent of hydrogen bonding decreases and as the aggregates reach a nanometer size in which interfacial effects distort the arrangement of an alcohol's hydrocarbon chain enough to decrease the relaxation time, its distribution, and the probability of the parallel correlation of dipoles.

The study raises the issue of how the dielectric relaxation times of the alcohol and isoamylbromide can be related to the macroscopic viscosity of the solution through the equality of the shear and dielectric relaxation times or else can be related to the continuum model high-frequency shear moduli of the clusters and of the liquid matrix. It is also difficult to see how this behavior may be interpreted in terms of the configurational entropy theory. ${ }^{53}$ It remains a challenge to address these issues in the future.

\section{ACKNOWLEDGMENT}

One of the authors (G.P.J.) would like to thank the hospitality of Trinity College, Dublin, for his stay during the period of this study. Post-doctoral Fellowship of G. P. in 2005-2006 was funded by Science Foundation of Ireland Grant SFI(02/In.1/I031).

${ }^{1}$ A. Schallamach, Trans. Faraday Soc. 42A, 180 (1946).

${ }^{2}$ D. J. Denney, J. Chem. Phys. 30, 1019 (1959).

${ }^{3}$ S. S. N. Murthy and M. Tyagi, J. Chem. Phys. 117, 3837 (2002).

${ }^{4} \mathrm{~J}$. Zarzycki, Glasses and the Vitreous State (Cambridge University Press, Cambridge, 1991).

${ }^{5}$ E. Donth, The Glass Transition: Relaxation Dynamics in Liquids and Disordered Materials (Springer-Verlag, Berlin, 2001).

${ }^{6}$ C. A. Angell, K. L. Ngai, G. B. McKenna, P. F. McMillan, and S. W. Martin, J. Appl. Phys. 88, 3113 (2000).

${ }^{7}$ R. Böhmer, G. Hinze, G. Diezemann, B. Geil, and H. Sillescu, Europhys. Lett. 36, 55 (1996).

${ }^{8}$ H. Sillescu, J. Non-Cryst. Solids 243, 81 (1999).

${ }^{9}$ H. Vogel, Phys. Z. 22, 645 (1921).

${ }^{10}$ G. S. Fulcher, J. Am. Chem. Soc. 8, 339 (1925).

${ }^{11}$ G. Tammann and W. Hesse, Z. Anorg. Allg. Chem. 156, 245 (1926).

${ }^{12}$ G. P. Johari, J. Chem. Phys. 58, 1766 (1973).

${ }^{13}$ G. P. Johari, Ann. N.Y. Acad. Sci. 279, 117 (1976).

${ }^{14}$ J. Y. Cavaille, J. Perez, and G. P. Johari, Phys. Rev. B 39, 2411 (1989).

${ }^{15}$ B. Bagchi, A. Chandra, and S. A. Rice, J. Chem. Phys. 93, 8991 (1990).

${ }^{16}$ K. L. Ngai, AIP Conf. Proc. 708, 515 (2004).

${ }^{17}$ G. P. Johari, G. Power, and J. K. Vij, J. Chem. Phys. 117, 1714 (2002).

${ }^{18}$ F. Scarponi, L. Comez, D. Fioretto, and L. Palmieri, Phys. Rev. B 70, 054203 (2004).

${ }^{19}$ M. L. Lind, G. Duan, and W. L. Johnson, Phys. Rev. Lett. 97, 015501 (2006).

${ }^{20}$ O. E. Kalinovskaya and J. K. Vij, J. Chem. Phys. 111, 10979 (1999).

${ }^{21}$ O. E. Kalinovskaya and J. K. Vij, J. Chem. Phys. 112, 3262 (2000).

${ }^{22}$ K. S. Cole and R. H. Cole, J. Chem. Phys. 9, 341 (1941).

${ }^{23}$ D. W. Davidson and R. H. Cole, J. Chem. Phys. 18, 1417 (1950).

${ }^{24}$ W. Huang and R. Richert, J. Chem. Phys. 124, 164510 (2006).

${ }^{25}$ Handbook of Chemistry and Physics, 62nd ed., edited by R. C. Weast (CRC, Boca Raton, 1982).

${ }^{26}$ L.-M. Wang, S. Shahriari, and R. Richert, J. Phys. Chem. B 109, 23255 (2005).

${ }^{27}$ J. B. Hasted, Aqueous Dielectrics (Chapman and Hall, London, 1973).

${ }^{28}$ A. Ansón, R. Garriga, S. Martínez, P. Pérez, and M. Gracia, J. Chem. Eng. Data 50, 1478 (2005).

${ }^{29}$ W. Gorbatschow, M. Arndt, R. Stannarius, and F. Kremer, Europhys. Lett. 35, 719 (1996).

${ }^{30}$ F. Kremer, A. Huwe, M. Arndt, P. Behrens, and W. Schwieger, J. Phys.: Condens. Matter 11, A175 (1999).

${ }^{31}$ O. Trofymluk, A. A. Levchenko, and A. Navrotsky, J. Chem. Phys. 123, 194509 (2005).

${ }^{32}$ L. Onsager, J. Am. Chem. Soc. 58, 1486 (1936). 
${ }^{33}$ J. G. Kirkwood, J. Chem. Phys. 4, 592 (1936).

${ }^{34}$ H. Fröhlich, Theory of Dielectrics, 2nd ed. (Oxford University Press, Oxford, 1958).

${ }^{35}$ W. Dannhauser, L. W. Bahe, R. Y. Lin, and A. F. Flueckinger, J. Chem. Phys. 43, 257 (1965).

${ }^{36}$ W. Dannhauser, J. Chem. Phys. 48, 1911 (1968); J. Chem. Phys. 48, 1918 (1968).

${ }^{37}$ G. P. Johari and W. Dannhauser, J. Phys. Chem. 48, 5114 (1968).

${ }^{38}$ K. Hofer and G. P. Johari, J. Chem. Phys. 95, 2020 (1991).

${ }^{39}$ C. Hansen, F. Stickel, T. Berger, R. Richert, and E. W. Fischer, J. Chem. Phys. 107, 1086 (1997)

${ }^{40}$ A. Kudlik, C. Tschirwitz, S. Benkhof, T. Blochowicz, and E. Rössler, Europhys. Lett. 40, 649 (1997).

${ }^{41}$ G. P. Johari, O. E. Kalinovskaya, and J. K. Vij, J. Chem. Phys. 114, 4634 (2001).
${ }^{42}$ O. E. Kalinovskaya, J. K. Vij, and G. P. Johari, J. Phys. Chem. A $\mathbf{1 0 5}$, 5061 (2001).

${ }^{43}$ G. Power, G. P. Johari, and J. K. Vij, J. Chem. Phys. 116, 4192 (2002).

${ }^{44}$ S. Schwerdtfeger, F. Köhler, R. Pottel, and U. Kaatze, J. Chem. Phys. 115, 4186 (2001).

${ }^{45}$ C. M. Roland, S. Hensel-Biewloka, M. Paluch, and R. Casalini, Rep. Prog. Phys. 68, 1405 (2005).

${ }^{46}$ G. P. Johari and O. Andersson, J. Chem. Phys. 125, 124501 (2006).

${ }^{47}$ L.-M. Wang, and R. Richert, J. Chem. Phys. 123, 054516 (2005).

${ }^{48}$ W. Oldekop, Glastech. Ber. 30, 8 (1957).

${ }^{49}$ W. T. Laughlin and D. R. Uhlmann, J. Phys. Chem. 76, 2317 (1972).

${ }^{50}$ J. E. Anderson and R. Ullman, J. Chem. Phys. 47, 2178 (1967).

${ }^{51}$ G. P. Johari and W. Dannhauser, J. Chem. Phys. 50, 1862 (1969)

${ }^{52}$ A. Patist, T. Axelberd, and D. O. Shah, J. Colloid Interface Sci. 208, 259 (1998).

${ }^{53}$ G. Adam and J. H. Gibbs, J. Chem. Phys. 43, 139 (1965). 